\title{
Axisymmetric Creeping Flow of a Micropolar Fluid over a Sphere Coated with a Thin Fluid Film
}

\author{
B. R. Gupta ${ }^{1}$ and S. Deo ${ }^{2 \dagger}$ \\ ${ }^{1}$ Department of mathematics, Jaypee University of Engineering \&Technology, Guna, MP, India \\ ${ }^{2}$ Department of mathematics, University of Allahabad, Allahabad-211002, UP, India
}

$\dagger$ †orresponding Author Email: sd_mathau@yahoo.co.in

(Received January 16, 2010; accepted July 21, 2012)

\begin{abstract}
Consideration is given to the problem of steady axisymmetric Stokes flow of a micropolar fluid past a sphere coated with a thin, immiscible Newtonian fluid layer. Inertial effects are neglected for both the outer fluid and the fluid film. The stream function solutions of the governing equations are obtained in terms of modified Bessel functions and Gegenbauer functions. The explicit expressions of flow fields are determined by applying the boundary conditions at the coated sphere interface and uniform velocity at infinity. The drag force experienced by the fluid-coated sphere is evaluated and its variation is studied with respect to various geometric and material parameters. It is found that a sphere without coating experience greater resistance in comparison to coated fluid. Some well-known results are then deduced from the present study.
\end{abstract}

Keywords: Micropolar fluid, Stokes flow, Modified Bessel functions, Drag force.

\section{NOMENCLATURE}

$D_{N}$
$F$
$G_{n}(\zeta) \& H_{n}(\zeta)$
$K_{n-1 / 2}(\lambda r) \&$
$I_{n-1 / 2}(\lambda r)$
$i$
$\ell=b / a$
$p^{(i)}$
$r$
$r, \theta, \varphi$
$T$
$\mathbf{v}^{(i)}$

\section{INTRODUCTION}

It has been recognized that the internal degrees of freedom of molecules play significant roles in determining equilibrium macroscopic or thermo dynamical properties of a substance. This has been less appreciated by phenomenologist as these same internal degrees of freedom may need to be recognized explicitly in the kinematical description of nonequilibrium process, such as momentum transport. The simplest theory considered for structured fluids, the theory of micropolar fluid introduced by Eringen (1964). There are some relevant previous investigations

$\alpha, \beta, \gamma$
$\varepsilon=a-b$
$\kappa$
$v_{\phi}$
$\theta$
$\mu$
$\psi^{(i)}$
$\omega$
2
1

Gyroviscosity coefficients thickness of coated fluid vortex viscosity coefficient mcro-rotation component

$\theta$-component viscosity coefficient stream functions

microrotation vector thin liquid coated region outer continuous region on creep flows in micropolar fluid mechanics as described nicely in the book written by Lukaszewicz (1999). Drag on an axially symmetric body in Stokes flow of a micropolar fluid has been evaluated by Ramkisoon and Majumdar (1976) and they observed that the drag in the micropolar fluid is greater than that in the classical fluid. The problems of the flow of a viscous fluid past a micropolar fluid sphere and the flow of a micropolar fluid past a viscous fluid drop have been discussed by Niefer and Kaloni (1980). Ramkissoon (1985) has obtained the solution for the problem of a micropolar fluid flow around a Newtonian 
fluid sphere and evaluated drag force exerted on the sphere. The Stokes flow problem of a viscous incompressible fluid past a fluid prolate spheroid parallel to its axis of revolution is investigated by Deo and Datta (2003) and evaluated the drag force experienced by fluid prolate spheroid. Recently, Gupta and Deo (2010) have studied Stokes flow of micropolar fluid past a porous sphere with non-zero boundary condition for micro rotations.

In recent years, the applications of compound multiphase drops, which are made of an outer liquid shell and an inner rigid sphere, gas bubble or immiscible liquid drop, have received significant interest. The case of creeping flow past bubbles and drops coated with thin films has been investigated by Sadhal and Johnson (1983). The drag coefficient of a spherical encapsulated liquid drop and an ensemble encapsulated liquid drop has been evaluated by Kawano and Hashimoto (1992) and Ferreira et al. (2003), respectively. Here, we have considered a variation of the classical unbounded problem by examining the incompressible low-Reynolds- number micropolar fluid flow past a rigid sphere having a thin layer of immiscible fluid covering its surface, i.e. a thin fluid film. Such flows for Newtonian fluid have been modeled earlier by Gupalo and Ryazantsev (1974), O'Neill and Ranger (1981), Johnson (1981), Kawano and Hashimoto (1997). Recently, a method was developed to solve the problem of an arbitrary Stokes flow of a viscous, incompressible fluid past a sphere with a thin coating of a fluid of a different viscosity by Choudhuri and Padmavati (2010).

The purpose of this study is to extend the work of Johnson (1981) by taking micropolar fluid in place of Newtonian fluid for outer region. This study undertakes the problem of steady axisymmetric Stokes flow of a micropolar fluid past a sphere coated with a thin, immiscible Newtonian fluid layer. Inertial effects are neglected for both the outer fluid and the thin fluid film. It is further assumed that the motion is sufficiently slow, the surface tension between fluids is very high and the fluid film coating is very thin, so that the shape of coating remains spherical. The stream function solutions of the governing equations are obtained in terms of modified Bessel functions and Gegenbauer functions. The explicit expressions of flow fields are determined by applying the boundary conditions at the coated sphere interface and uniform velocity at infinity. The drag force experienced by a fluid-coated sphere is evaluated and its variation is studied with respect to various geometric and material parameters. Some wellknown results are then deduced from the present analysis.

\section{MATHEMATICAL Formulation}

Here, we have considered a rigid sphere of radius $b$ coated with a thin Newtonian fluid film of radius $a$ $(a>b)$ in an unbounded medium with origin at the centre $O$ of the sphere (Fig.1). We assume that the coated sphere is stationary and a steady axisymetric creeping flow of a micropolar fluid has been established around it by a uniform far-field flow with velocity of magnitude $\mathrm{U}$ directed along z-axis.

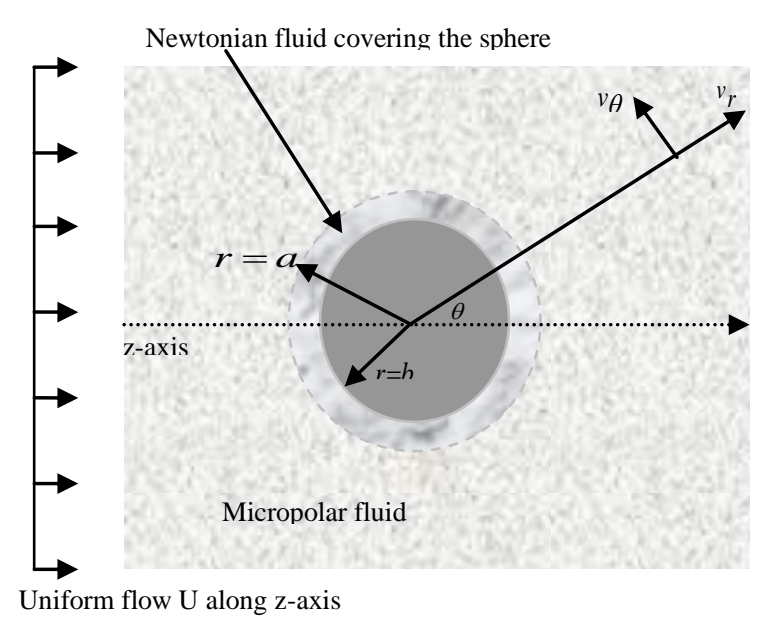

Fig. 1. Schematic representation of flow

Let the index in the superscripts under bracket of an entity $\chi^{(i)}, i=1,2$ indicates the outside and inside regions of the coated fluid, respectively. The general form of governing equations for the slow steady motion of micropolar fluids under Stokes approximation for outside region can be written as follows

$$
\begin{aligned}
& \operatorname{div} \mathbf{v}^{(1)}=0 \\
& -\nabla p^{(1)}+\kappa \nabla \times \boldsymbol{\omega}-\left(\mu_{1}+\kappa\right) \nabla \times \nabla \times \mathbf{v}^{(1)}=0 \\
& -2 \kappa \boldsymbol{\omega}+\kappa \nabla \times \mathbf{v}^{(1)}-\gamma \nabla \times \nabla \times \boldsymbol{\omega} \\
& +(\alpha+\beta+\gamma) \nabla(\nabla . \boldsymbol{\omega})=0
\end{aligned}
$$

For region (2), occupied by Newtonian fluid film ( $b \leq r \leq a)$, we use the continuity equation and Stokes approximation of momentum equation

$\operatorname{div} \mathbf{v}^{(2)}=0$

$\mu_{2} \nabla^{2} \mathbf{v}^{(2)}=\nabla p^{(2)}$

where, $\mathbf{v}^{(i)}$ being the velocity vectors, $p^{(i)}$ the pressures, $\omega=v_{\phi}(r, \theta) \hat{e}_{\phi}$ the microrotation vector, $\mu_{i}$ are classical viscosity coefficient of fluids, $\kappa, \lambda$ the vortex viscosity coefficients, $\alpha, \beta, \gamma$ are gyroviscosity coefficients satisfying the following inequalities

$$
\begin{aligned}
& 3 \alpha+\beta+\gamma \geq 0, \quad 2 \mu_{1}+\kappa \geq 0, \gamma \geq|\beta|, \quad \kappa \geq 0, \\
& \gamma \geq 0
\end{aligned}
$$

To non- dimensionalize the equations and variables, we put

$$
r=a \tilde{r}, \psi^{(i)}=U a^{2} \tilde{\psi}^{(i)}, p^{(i)}=\frac{\mu U}{a} \tilde{p}^{(i)},
$$$$
v_{\phi}^{(i)}=\frac{U}{a} \tilde{v}_{\phi}^{(i)}
$$

and dropping tildes subsequently in further analysis.

Since the flow field is axisymetric, we can introduce stream functions $\psi^{(i)}(r, \theta)$ which satisfy the continuity equations and related to the velocity in spherical coordinate system $(r, \theta, \phi)$ by (Happel and Brenner 1983) 


$$
\begin{aligned}
& \mathbf{v}^{(i)}=v_{r}^{(i)}(r, \theta) \hat{e}_{r}+v_{\theta}^{(i)}(r, \theta) \hat{e}_{\theta}= \\
& -\operatorname{curl}\left[\frac{\psi^{(i)}}{r \sin \theta} \hat{e}_{\varphi}\right]
\end{aligned}
$$

and obtain two velocity components of the flow in two regions $i=1,2$ as

$$
\left.\begin{array}{c}
v_{r}^{(i)}=-\frac{1}{r^{2} \sin \theta} \frac{\partial \psi^{(i)}}{\partial \theta} \\
v_{\theta}^{(i)}=\frac{1}{r \sin \theta} \frac{\partial \psi^{(i)}}{\partial r}
\end{array}\right\}
$$

Eliminating pressures from Eq. (2) and Eq. (5) which provides the following differential equations to be solved for stream functions, respectively as

$$
\begin{aligned}
& E^{4}\left(E^{2}-\lambda^{2}\right) \psi^{(1)}=0 \\
& E^{4} \psi^{(2)}=0
\end{aligned}
$$

Similarly, from Eq. (3), we can get

$v_{\phi}=\frac{1}{2 r \sin \theta}\left[E^{2} \psi^{(1)}+\frac{\gamma\left(\mu_{1}+\kappa\right)}{\kappa^{2}} E^{4} \psi^{(1)}\right]$

where $E^{2}=\frac{\partial^{2}}{\partial r^{2}}+\frac{\left(1-\zeta^{2}\right)}{r^{2}} \frac{\partial^{2}}{\partial \zeta^{2}}, \zeta=\cos \theta$

micropolar parameter

$$
\lambda^{2}=\frac{\kappa\left(2 \mu_{1}+\kappa\right)}{\gamma\left(\mu_{1}+\kappa\right)} a^{2}
$$

\section{Method Of Solution}

The solution of Eq. (10) and Eq. (11) can be obtained by superimposing the solutions of

$$
\begin{aligned}
& E^{2} \psi_{0}=0 \\
& E^{4} \psi_{1}=0 \\
& \left(E^{2}-\lambda^{2}\right) \psi_{2}=0
\end{aligned}
$$

in the form as

$$
\psi^{(1)}=\psi_{0}+\psi_{1}+\psi_{2}
$$

and

$$
\psi^{(2)}=\psi_{0}+\psi_{1}
$$

respectively.

Using the method of separation of variables, the solutions of Eqs. (14), (15) and (16) can be obtained, respectively as

$$
\begin{aligned}
\psi_{0} & =\sum\left[A_{n} r^{n}+B_{n} r^{-n+1}\right]\left(A_{n}^{\prime} G_{n}(\zeta)+B_{n}^{\prime} H_{n}(\zeta)\right) \\
\psi_{1} & =\sum\left[C_{n} r^{n+2}+D_{n} r^{-n+3}\right] \\
& \left(C_{n}^{\prime} G_{n}(\zeta)+D_{n}^{\prime} H_{n}(\zeta)\right]
\end{aligned}
$$

$$
\begin{aligned}
& \psi_{2}=\sum {\left[E_{n} \sqrt{r} K_{n-1 / 2}(\lambda r)+F_{n} \sqrt{r} I_{n-1 / 2}(\lambda r)\right] } \\
& \times\left(E_{n}^{\prime} G_{n}(\zeta)+F_{n}^{\prime} H_{n}(\zeta)\right),
\end{aligned}
$$

where, $K_{n-1 / 2}(\lambda r) \quad$ and $\quad I_{n-1 / 2}(\lambda r) \quad$ are modified Bessel functions and $G_{n}(\zeta), H_{n}(\zeta)$ are Gegenbauer functions of first and second kinds, respectively. Due to irregularity of $G_{0}(\zeta), G_{1}(\zeta)$ and $H_{n}(\zeta)$ at the symmetry axis-z, we have ignored the terms which are multiplied by $G_{0}(\zeta), G_{1}(\zeta)$ and $H_{n}(\zeta)$ for all values of $\mathrm{n}$ (Abramowitz \& Stegun 1970).

Therefore, the complete regular solution of Eqs.(10) and (11) takes the following forms, respectively as

$$
\begin{array}{r}
{\left[A_{n} r^{1-n}+B_{n} r^{n}+C_{n} r^{3-n}+\right.} \\
\psi^{(1)}=\sum_{n=2}^{\infty} D_{n} r^{n+2}+E_{n} \sqrt{r} K_{n-\frac{1}{2}}(\lambda r) \\
\left.+F_{n} \sqrt{r} I_{n-\frac{1}{2}}(\lambda r)\right] G_{n}(\varsigma)
\end{array}
$$

Using regularity condition at infinity, we notice that the terms involving $r^{n}, \quad r^{n+2}$ and $I_{n-1 / 2}(\lambda r)$ must be absent from Eq. (22) with the exception of the term $r^{2}$. In order to satisfy the spherical far field condition for the free flow, we are forced to retain only the term of order $n=2$ of the general solutions for the free flow stream function. Consequently, stream function reduces to

$$
\begin{gathered}
\begin{aligned}
& \psi^{(1)}(r, \zeta)=\left[A_{2} r^{-1}+B_{2} r^{2}+C_{2} r\right. \\
&+\left.E_{2} \sqrt{r} K_{3 / 2}(\lambda r)\right] G_{2}(\zeta), \\
& \psi^{(2)}(r, \zeta)=\left[A_{2}^{*} r^{-1}+B_{2}^{*} r^{2}+C_{2}^{*} r+D_{2}^{*} r^{4}\right] G_{2}(\zeta)
\end{aligned}
\end{gathered}
$$

Substituting the value of $\psi^{(1)}$ in Eq. (12), we get the microrotation component as

$$
\begin{aligned}
v_{\phi}(r, \zeta)= & \frac{1}{r} \sin \theta \\
& \left.\times E_{2} \sqrt{r} K_{3 / 2}(\lambda r)\right] G_{2}(\zeta) .
\end{aligned}
$$

\subsection{Boundary Conditions}

The boundary conditions to be satisfied at the surface of the fluid sphere which are physically realistic and mathematically consistent for this proposed problem can be taken as:

I. The kinematical condition of mutual impenetrability at the surface $r=a$ requires that

$$
\begin{aligned}
& \psi^{(1)}(r, \zeta)=0 \\
& \psi^{(2)}(r, \zeta)=0
\end{aligned}
$$


B. R. Gupta et al. / JAFM, Vol. 6, No. 2, pp. 149-155, 2013.

II. The continuity of tangential velocity across the surface implies that

$$
\frac{\partial \psi^{(1)}(r, \zeta)}{\partial r}=\frac{\partial \psi^{(2)}(r, \zeta)}{\partial r} \text { at } r=a
$$

III. We assume that tangential components of stresses are continuous across the surface, so that we may take $T_{r \theta}^{(1)}=T_{r \theta}^{(2)}$ at $r=a$, which on simplification provides

$$
\begin{aligned}
& -\left(2 \mu_{1}+\kappa\right) \frac{\partial \psi^{(1)}}{\partial r}+\left(\mu_{1}+\kappa\right) \frac{\partial^{2} \psi^{(1)}}{\partial r^{2}} \\
& =-2 \mu_{2} \frac{\partial \psi^{(2)}}{\partial r}+\mu_{2} \frac{\partial^{2} \psi^{(2)}}{\partial r^{2}} .
\end{aligned}
$$

IV. Next, we utilize the no-spin condition for microrotation on the boundary of the coated sphere i.e.

$$
v_{\phi}=0 \text { at } r=a
$$

V. On the inner solid sphere $r=b$, the conditions of impenetrability and no- slip provides

$$
\begin{aligned}
& \psi^{(2)}(r, \zeta)=0 \\
& \psi_{r}^{(2)}(r, \zeta)=0
\end{aligned}
$$

VI. The condition at infinity for uniform stream i.e.

$$
\psi^{(1)} \rightarrow \frac{1}{2} r^{2} \sin ^{2} \theta \text { as } r \rightarrow \infty \text { gives } B_{2}=1
$$

\subsection{Determination of arbitrary constants}

Applying these above boundary conditions (Eqs. (27) (34)), we obtain

$$
\begin{aligned}
& 1+A_{2}+C_{2}+E_{2} K_{3 / 2}(\lambda)=0 \\
& A_{2}^{*}+B_{2}^{*}+C_{2}^{*}+D_{2}^{*}=0 \\
& C_{2}-A_{2}+E_{2} K^{\prime} 3 / 2(\lambda)+A_{2}^{*}-2 B_{2}^{*} \\
& -C_{2}^{*}-4 D_{2}^{*}=-2 \\
& -2 C_{2}+\frac{2 \lambda^{2}\left(\mu_{1}+\kappa\right)}{\kappa} E_{2}=0 \\
& \left(4 \mu_{1}+3 \kappa\right) A_{2}-\left(2 \mu_{1}+\kappa\right) C_{2}+E_{2}\left(\left\{\left(4 \mu_{1}+3 \kappa\right)\right.\right. \\
& \left.\left.+\lambda^{2}\left(\mu_{1}+\kappa\right)\right\} K_{3 / 2}(\lambda)+\left(2 \mu_{1}+\kappa\right) \lambda K_{1 / 2}(\lambda)\right) \\
& -4 \mu_{2} A_{2}^{*}+2 \mu_{2} B_{2}^{*}+2 \mu_{2} C_{2}^{*}-4 \mu_{2} D_{2}^{*}=2 \mu_{1}, \\
& A_{2}^{*} \ell^{-1}+B_{2}^{*} \ell^{2}+C_{2}^{*} \ell+D_{2}^{*} \ell^{4}=0 \\
& -A_{2}^{*} \ell^{-2}+2 B_{2}^{*} \ell+C_{2}^{*}+4 D_{2}^{*} \ell^{3}=0
\end{aligned}
$$

Solving these above Eqs. (35) to (41), we found that

$$
\begin{aligned}
& A_{2}=\left[-\lambda \kappa\left[\left(4+3 l-3 l^{2}-4 l^{3}\right)\left(k+2 \mu_{1}\right)+\right.\right. \\
& \left.6\left(2+3 l+3 l^{2}+2 l^{3}\right) \mu_{2}\right] K_{1 / 2}(\lambda)+ \\
& 3\left[\kappa\left(4+3 l-3 l^{2}-4 l^{3}\right)\left(\kappa+2 \mu_{1}\right)+2 .\right. \\
& \left(2+3 l+3 l^{2}+2 l^{3}\right)\left\{\lambda^{2} \mu_{1} \mu_{2}+\right. \\
& \left.\left.\left(3+\lambda^{2}\right) \kappa \mu_{2}\right] K_{1 / 2}(\lambda)\right] /-\lambda \Delta,
\end{aligned}
$$$$
C_{2}=3 \lambda\left(\kappa+\mu_{1}\right)\left[\left(4+3 l-3 l^{2}-4 l^{3}\right)\right.
$$$$
\left(\kappa+2 \mu_{1}\right)+6\left(2+3 l+3 l^{2}+2 l^{3}\right)
$$$$
\left.\mu_{2}\right] K_{3 / 2}(\lambda) /-\Delta
$$

$$
\begin{aligned}
E_{2}= & 3 \kappa\left[\left(4+3 \ell-3 \ell^{2}-4 \ell^{3}\right)\left(\kappa+2 \mu_{1}\right)\right. \\
& \left.+6\left(2+3 \ell+3 \ell^{2}+2 \ell^{3}\right) \mu_{2}\right](\ell-1) /-\lambda \Delta,
\end{aligned}
$$

$A_{2}^{*}=3 l^{3}(1+2 l) \lambda\left(\kappa^{2}+3 \kappa \mu_{1}+2 \mu_{1}^{2}\right)$

$K_{3 / 2}(\lambda) /(l-1) \Delta$,

$B_{2}^{*}=3\left(2+4 l+6 l^{2}+3 l^{3}\right) \lambda\left(\kappa^{2}+3 \kappa \mu_{1}+2 \mu_{1}^{2}\right)$

$\times K_{3 / 2}(\lambda) /(l-1) \Delta$,

$C_{2}^{*}=-3 l\left(3+6 l+4 l^{2}+2 l^{3}\right)$

$\lambda\left(\kappa^{2}+3 \kappa \mu_{1}+2 \mu_{1}^{2}\right)$

$\times K_{3 / 2}(\lambda) /(l-1) \Delta$,

$D_{2}^{*}=-3(l+2) \lambda\left(\kappa^{2}+3 \kappa \mu_{1}+2 \mu_{1}^{2}\right)$

$K_{3 / 2}(\lambda) /(l-1) \Delta$,

where

$$
\begin{aligned}
& \Delta=\kappa\left[\left(\kappa+2 \mu_{1}\right)\left(-4-3 l+3 l^{2}+4 l^{3}\right)\right. \\
& \left.-6\left(2+3 l+3 l^{2}+2 l^{3}\right) \mu_{2}\right] K_{1 / 2}(\lambda)- \\
& 3 \lambda\left(\kappa+\mu_{1}\right)\left[\left(\kappa+2 \mu_{1}\right) \times\left(-4-3 l+3 l^{2}+4 l^{3}\right)\right. \\
& \left.-4\left(2+3 l+3 l^{2}+2 l^{3}\right) \mu_{2}\right] \times K_{3 / 2}(\lambda)
\end{aligned}
$$

We have thus determined the explicit expressions of stream functions (Eqs. (24) and (25)) for the flow, as all constants have been determined.

\section{Evaluation Of Drag Force}

In order to determine the hydrodynamic drag force $F$ acting on the coated sphere, which will be directed along the symmetrical axis, we need to evaluate the integral

$$
F=2 \pi a^{2} \int_{0}^{\pi}\left(T_{r r}^{(1)} \cos \theta-T_{r \theta}^{(1)} \sin \theta\right)_{r=a} \sin \theta d \theta
$$

Putting the expressions for the stresses in spherical polar coordinates in Eq. (50) and evaluating the integral, we find that 


$$
\begin{aligned}
& F=2 \pi\left(2 \mu_{1}+\kappa\right) a U C_{2} \\
& =6 \pi\left(2 \mu_{1}+\kappa\right) a U \lambda\left(\kappa+\mu_{1}\right)\left[\left(4+3 l-3 l^{2}-4 l^{3}\right)\right. \\
& \left.\left(\kappa+2 \mu_{1}\right)+6\left(2+3 l+3 l^{2}+2 l^{3}\right) \mu_{2}\right] \\
& K_{3 / 2}(\lambda) /-\Delta
\end{aligned}
$$

where $\Delta$ is given by Eq. (49).

\section{RESUlt AND Discussion}

At the outset, it is instructive to consider some limiting situations of the drag force as discussed below:

Drag on a Newtonian fluid sphere in the micropolar fluid $(b \rightarrow 0)$ :

When $b=0$, i.e., $\ell=b / a=0$, then coated fluid sphere behaves like a fluid sphere of radius $a$. In this case, we get the value of $C_{2}$ from Eq. (43) as

$$
\begin{aligned}
& C_{2}=\left[3 \lambda\left(\mu_{1}+\kappa\right)\left(3 \mu_{2}+2 \mu_{1}+\kappa\right) K_{3 / 2}(\lambda)\right] \\
& /\left[\kappa\left(3 \mu_{2}+2 \mu_{1}+\kappa\right) K_{1 / 2}(\lambda)-3 \lambda\left(\mu_{1}+\kappa\right)\right. \\
& \left.\times\left(2 \mu_{2}+2 \mu_{1}+\kappa\right) K_{3 / 2}(\lambda)\right] .
\end{aligned}
$$

Thus the value of drag force, from the Eq. (51), experienced by the Newtonian fluid sphere comes out as

$$
F=\frac{6 \pi\left(2 \mu_{1}+\kappa\right) a U \lambda\left(\mu_{1}+\kappa\right)\left(3 \mu_{2}+2 \mu_{1}+\kappa\right) K_{3 / 2}(\lambda)}{\kappa\left(3 \mu_{2}+2 \mu_{1}+\kappa\right) K_{1 / 2}(\lambda)-3 \lambda\left(\mu_{1}+\kappa\right)\left(2 \mu_{2}+2 \mu_{1}+\kappa\right) K_{3 / 2}(\lambda)}
$$

A known result for the drag force has been reported earlier by Ramkissoon (1985).

Drag on a rigid sphere in an unbounded micropolar fluid $(b \rightarrow a)$ :

If $b=a$, i.e., $\ell=b / a=1$, then coated fluid sphere will become a rigid sphere of radius $a$. In this case, we get the value of the constant $C_{2}$ from Eq. (43) as

$$
C_{2}=-\frac{3 \lambda\left(\mu_{1}+\kappa\right) K_{3 / 2}(\lambda)}{\kappa K_{1 / 2}(\lambda)+2 \lambda\left(\mu_{1}+\kappa\right) K_{3 / 2}(\lambda)}
$$

Thus the value of drag force, from the Eq. (51), experienced by the rigid sphere in an unbounded micropolar fluid comes out as

$$
F=-\frac{6 \pi a U\left(2 \mu_{1}+\kappa\right) \lambda\left(\mu_{1}+\kappa\right) K_{3 / 2}(\lambda)}{\kappa K_{1 / 2}(\lambda)+2 \lambda\left(\mu_{1}+\kappa\right) K_{3 / 2}(\lambda)}
$$

A known result for the drag has been reported earlier by Ramkissoon and majumdar (1976).

Stokes flow past a Newtonian fluid sphere in an unbounded Newtonian fluid $(\kappa \rightarrow o, b \rightarrow 0)$ :

In this case, the value of drag force from the Eq. (53) by applying the limit $\kappa \rightarrow o$ reduces to

$$
F=-6 \pi \mu_{1} U a \frac{1+\frac{2}{3} \sigma}{1+\sigma}, \sigma=\frac{\mu_{1}}{\mu_{2}}
$$

This result has been reported in the book of Happel and Brenner (1983).

\section{The dimensionless drag coefficient:}

The dimensionless drag coefficient is defined as

$$
D_{N}=F /\left(-4 \pi \mu_{1} U a\right)
$$

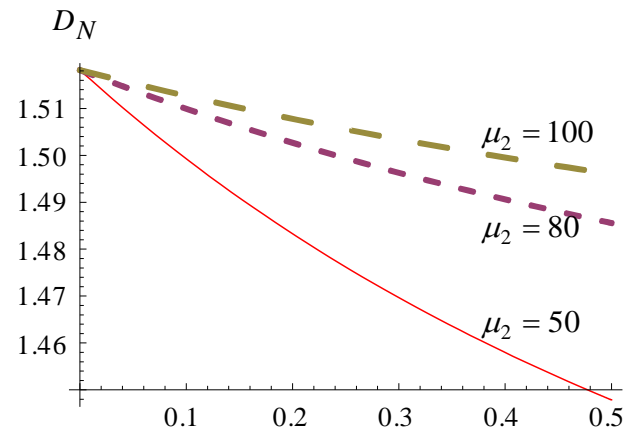

Fig. 2. Effect of thickness $\varepsilon$ on dimensionless drag $D_{N}$ for different values of $\mu_{2}$ when $\mu_{1}=10$ and $\kappa=0.5$

The dependence of the dimensionless drag $D_{N}$ on thickness $\varepsilon=a(1-\ell)$ of fluid coating is depicted in Fig. 2 for $\mu_{1}=10, \kappa=0.5$ and $\mu_{2}=50,80,100$. The drag coefficient $D_{N}$ attains maximum value at $\epsilon=0$ and decreases as $\epsilon$ increases. This physically means that a sphere without fluid coating experiences more drag while the presence of fluid coating reduces the drag on the sphere. Johnson (1981) had also found that the drag on a sphere is reduced by the presence of a fluid coating. This agrees with our outcome. In Fig. 3 the dimensionless drag $D_{N}$ is plotted against viscosity $\mu_{1}$ for $\mu_{2}=100, \epsilon=0.1$ and $\kappa=0.5,1.0,1.5$. It is seen that drag decreases as $\mu_{1}$ increases.

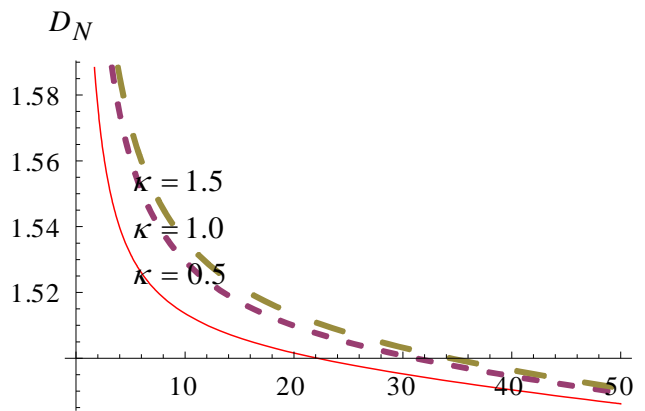

Fig. 3. Variation of dimensionless drag $D_{N}$ versus viscosity coefficient $\mu_{1}$ at $\varepsilon=0.1, \mu_{2}=100$ for various values of $\kappa$ 


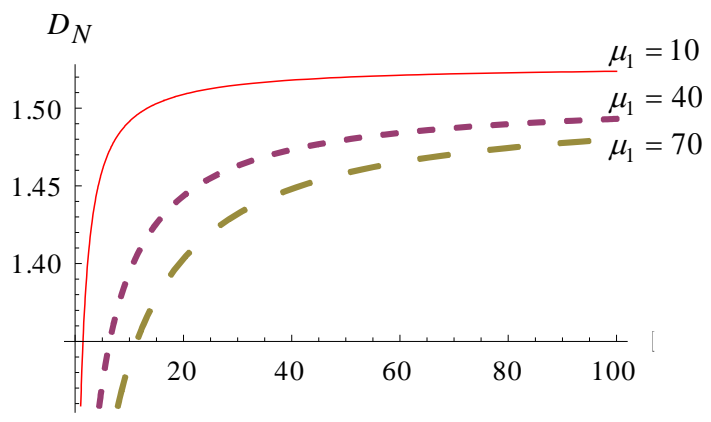

Fig. 4. Dependence of the dimensionless drag $D_{N}$ on viscosity coefficient $\mu_{2}$ for various values of $\mu_{1}$ at $\kappa=1.0$ and $\varepsilon=0.1$

The effect of viscosity $\mu_{2}$ of coated fluid on dimensionless drag $D_{N}$ is shown in Fig.4. For fixed values of $\epsilon, \kappa$ and $\mu_{1}$, the drag increases rapidly when $\mu_{1}>\mu_{2}$, but in case of $\mu_{1}<\mu_{2}$ the growth in drag is slow. A plot of dimensionless drag $D_{N}$ versus vortex viscosity $\kappa$ for fixed values of $\epsilon, \mu_{1}$ and $\mu_{2}$ is given by Fig.5. It is apparent that the small values of $\kappa$ correspond to a weak drag and as expected drag increases as $\kappa$ increases. This in turn shows that the drag in micropolar fluid is greater than that in the Newtonian fluid.

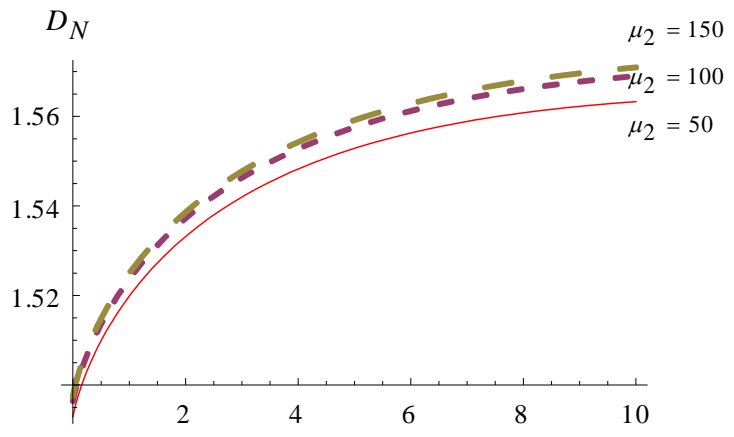

Fig. 5. Dependence of the dimensionless drag $D_{N}$ on vortex viscosity coefficient $\kappa$ for various values of $\mu_{2}$ at $\mu_{1=} 10$ and $\varepsilon=0.1$

\section{CONCLUISSION}

The solution to field equations are presented in terms of stream functions for steady axisymmetric Stokes flow of a micropolar fluid past a sphere coated with a thin, immiscible Newtonian fluid. A number of useful results are obtained from the solution, particularly the closedform expression for the drag force, the dependence of dimensionless drag coefficient on various fluid parameters. Since the viscosity and radius of coated fluid is taken to be completely arbitrary, most of the results apply to both rigid and fluid spheres. The present model is found to reduce to some well-known results from the literature in the limiting situations. It is interesting that a sphere without coating experience greater resistance in comparison to coated fluid. Also, an increase in vortex viscosity of micropolar fluid enhances the drag experienced by the coated sphere.

\section{REFERENCES}

Abramowitz, M and I. A. Stegun (1970).Handbook of Mathematical Functions. Dover Publications.

Choudhuri, D. and B. S. Padmavati (2010).A study of an arbitrary Stokes flow past a fluid coated sphere in a fluid of a different viscosity. Zeitschrift fü rangew and te Mathematik und Physik61(2), 317-328.

Deo, S. and S. Datta (2003). Stokes flow past a prolate fluid spheroid. Indian Journal of Pure and Applied Mathematics 34(5), 755-764.

Eringen, A.C. (1966). Theory of micropolar fluids. $J$. Math. Mech. 16(1), 1-18.

Gupalo, P.Yu. And Y. C.Ryazantsev (1974). Flow past a sphere coated by a liquid film for small Reynolds numbers. Fluid Dynamics9(5), 673682.

Gupta, B.R. and S. Deo (2010).Stokes flow of micropolar fluid past a porous sphere with nonzero boundary condition for microrotations. International Journal of Fluid MechanicsResearch37(5), 424-434.

Happel J and H. Brenner (1983).Low Reynolds Number Hydrodynamics. Martinus Nijoff Publishers, Hague.

Johnson, R. E. (1981). Stokes flow past a sphere coated with a thin fluid film. Journal of Fluid Mechanics 110, 217-238.

Kawano, S. and H. Hashimoto (1997). A numerical study on motion of a sphere coated with a thin film at intermediate Reynolds number. Journal of Fluids Engineering. ASME 119(2), 397-403.

Lukaszewicz, G. (1999). Micropolar Fluids- Theory and Applications, Birkhauser, Boston.

Niefer, R. and P. N. Kaloni (1980). On the motion of a micropolar fluid drop in a viscous fluid. Journal of Engineering Mathematics 14 (2), 107-116.

O'Neill, M.E. and K. B. Ranger (1981). On the slow motion of fluid coated sphere in an immiscible viscous fluid. C. R. Math. Rep. Acad. Sci. Can. 3(5), 261-266.

Ramkissoon, H. and S. R. Majumadar (1976). Drag on axially symmetric body in the Stokes flow of micropolar fluid. Physics of Fluids 19(1), 1621.

Ramkissoon, H. (1985). Flow of micropolar fluid past a Newtonian fluid sphere. Journal of Applied Mathematics and Mechanics / Zeitschrift für Angew and te Mathematik und Mechanik65(12), 635-637. 
B. R. Gupta et al. / JAFM, Vol. 6, No. 2, pp. 149-155, 2013.

Ramkissoon, H. (1986). Stokes flow past a slightly deformed fluid and sphere. Zeitschrift für angew and te Mathematik und Physik ZAMP 37(6), 859-866.
Sadhal S.S. and R. E. Johnson (1983). Stokes flow past bubbles and drops partially coated with thin films. Part 1.Stagnant cap of surfactant film exact solution. Journal of Fluid Mechanics.126, 237-250. 